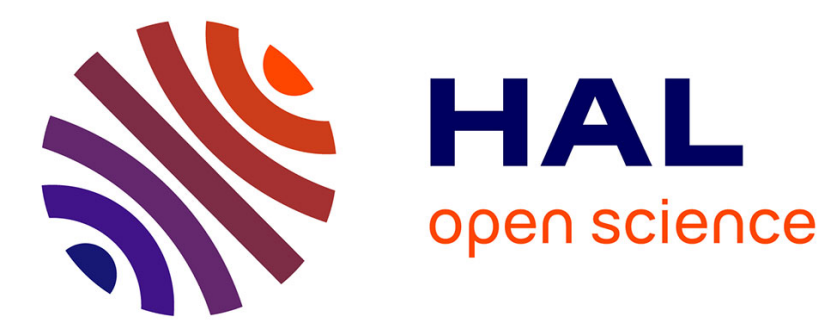

\title{
Cooperative Aerial Tele-Manipulation with Haptic Feedback
}

\author{
Mostafa Mohammadi, Antonio Franchi, Davide Barcelli, Domenico \\ Prattichizzo
}

\section{- To cite this version:}

Mostafa Mohammadi, Antonio Franchi, Davide Barcelli, Domenico Prattichizzo. Cooperative Aerial Tele-Manipulation with Haptic Feedback. IEEE/RSJ International Conference on Intelligent Robots and Systems (IROS 2016), Oct 2016, Daejeon, South Korea. hal-01348539v2

\section{HAL Id: hal-01348539 \\ https://hal.science/hal-01348539v2}

Submitted on 27 Jul 2016

HAL is a multi-disciplinary open access archive for the deposit and dissemination of scientific research documents, whether they are published or not. The documents may come from teaching and research institutions in France or abroad, or from public or private research centers.
L'archive ouverte pluridisciplinaire HAL, est destinée au dépôt et à la diffusion de documents scientifiques de niveau recherche, publiés ou non, émanant des établissements d'enseignement et de recherche français ou étrangers, des laboratoires publics ou privés. 


\title{
Cooperative Aerial Tele-Manipulation with Haptic Feedback
}

\author{
Mostafa Mohammadi ${ }^{1,2}$, Antonio Franchi ${ }^{3}$, Davide Barcelli ${ }^{1}$, and Domenico Prattichizzo ${ }^{1,2}$
}

\begin{abstract}
In this paper, we propose a bilateral tele-operation scheme for cooperative aerial manipulation in which a human operator drives a team of Vertical Take-Off and Landing (VTOL) aerial vehicles, that grasped an object beforehand, and receives a force feedback depending on the states of the system. For application scenarios in which dexterous manipulation by each robot is not necessary, we propose using a rigid tool attached to the vehicle through a passive spherical joint, equipped with a simple adhesive mechanism at the tool-tip that can stick to the grasped object. Having more than two robots, we use the extra degrees of freedom to find the optimal force allocation in term of minimum power and forces smoothness. The human operator commands a desired trajectory for the robot team through a haptic interface to a pose controller, and the output of the pose controller along with system constraints, e.g., VTOL limited forces and contact maintenance, defines the feasible set of forces. Then, an on-line optimization allocates forces by minimizing a cost function of forces and their variation. Finally, propeller thrusts are computed by a dedicated attitude and thrust controller in a decentralized fashion. Human/Hardware in the loop simulation study shows efficiency of the proposed scheme, and the importance of haptic feedback to achieve a better performance.
\end{abstract}

\section{INTRODUCTION}

In the last decade, small size Vertical Take-Off and Landing (VTOL) unmanned aerial vehicles have been a fundamental research topic in the robotics and control domains. In particular, quadrotors have been widely used for aerial photography, surveillance, and inspection. However, the potential capability of VTOLs is beyond the above mentioned sensing oriented applications; they can be utilized for aerial transportation [1], or to act on their environment using passive tools [2], [3], or using robotic manipulator installed on them [4].

The agility and high maneuverability of VTOLs make them appealing to be used also in search and rescue missions, transportation, and aerial manipulation using light-weight robotic manipulators [5]. For small size VTOLs the trade off between agility and ability to carry the large and heavy objects suggests a team of aerial robots. Regarding the mechanism, cooperative object transportation, grasping, and manipulation can be categorized into: without tool [6]-[8], with rigidly attached passive tools [9], [10], with ropes [11], [12], and with robotic manipulators [13].

Fully autonomous team control suffers from poor problem solving capabilities and inadequacy to unexpected environmental changes [14], and open loop human control leads

\footnotetext{
${ }^{1}$ Department of Information Engineering and Mathematics, University of Siena, via Roma 56, 53100 Siena, Italy mohammadi, barcelli,prattichizzodii.unisi.it

${ }^{2}$ Department of Advanced Robotics, Istituto Italiano di Tecnologia, via Morego 30, 16163 Genova, Italy mohammadi, prattichizzo@iit.it

${ }^{3}$ LAAS-CNRS, Université de Toulouse, CNRS, Toulouse, France, afranchilaas.fr
}

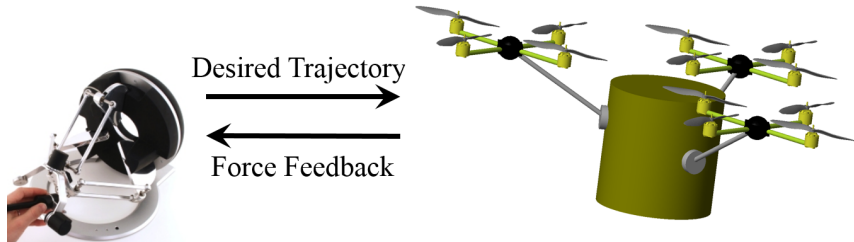

Fig. 1: Schematic representation of the proposed bilateral teleoperation for cooperative aerial manipulation.

to limited operator confidence [15], hence feeding back meaningful system information via a haptic device would improve performance.

In this paper, we propose to the best of our knowledge the first bilateral tele-operation scheme for cooperative aerial manipulation in which a human operator drives a team of VTOLs (as, e.g., quadrotors), that grasped an object beforehand, and receives a haptic feedback depending on the system's states, i.e., inertia of the object, and repulsive force to avoid obstacles. For application scenarios in which dexterous manipulation performed by a single Unmanned Aerial Vehicle (UAV) is not necessary, we propose using a rigid tool equipped with a simple gripping mechanism at the tool-tip that can stick to the grasped object as, e.g., a simple 1-DoF gripper, and attached to the VTOL Center of Mass (CoM) through a passive spherical joint. In fact, using a spherical joint at the CoM of the VTOL makes the fast rotational dynamics of the VTOLs decoupled from the slow dynamics of the grasped object [8]. From the point of view of the object dynamics, for more than two VTOLs we obtain a redundant system. These extra degrees of freedom in determining the forces applied by each VTOL is used to satisfy the constraints of the system. For each VTOL, in order to produce the desired 3D force, an appropriate total thrust and desired orientation is obtained.

The VTOL's passive tools need to be connected to the manipulated object. There are several possible approaches to realize it, such as the gripping mechanisms proposed in [16], small flat permanent magnets for ferromagnetic objects [9], vacuum suction cups [17], new technologies such as magnetorheological fluids [18], micro-structured adhesives [19], or dry adhesion materials [20]. Throughout the paper, we assume that one of these approaches is used, and we refer to the force produced at the contact points as the adhesive forces. We then explicitly take into account the constraints imposed by the maintenance of the adhesive force.

The rest of the paper is organized as follows. Section II explains the dynamic model of the system. Section III describes the control system architecture. Human/Hardwarein-the-loop simulation results in which a human operator drives a VTOL team, consisting of three quadrotors carrying 


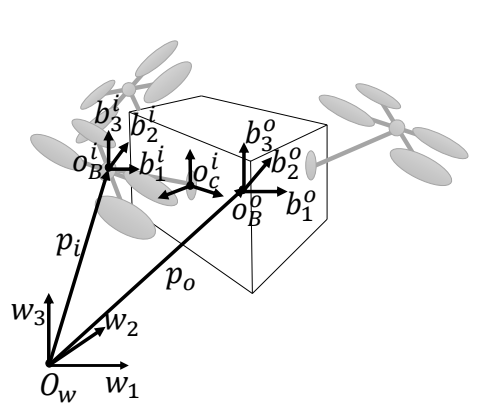

(a)

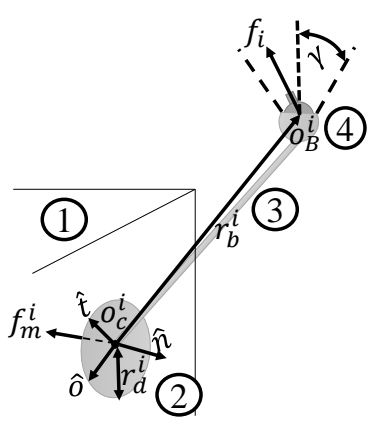

(b)
Fig. 2: $(a, b)$ Coordinate frames for a team of quadrotors manipulating an object. (b) Passive tool mechanism: an adhesive disc (2) attached to the grasped object (1) and connected to the spherical joint (4) through a lightweight bar (3).

an object, via a multi-DoF haptic device is presented in Section IV, and the concluding remarks are outlined in Section V.

\section{System MODEL}

The teleoperation scheme consists of a haptic device (master), a team of $n$ VTOL aerial robots, having physical constraints, attached to the object (slave), and a communication channel between them.

\section{A. Slave Model}

In order to derive the dynamic model of the system let us define the world frame $\mathscr{W}:\left\{O_{\mathscr{W}}, \boldsymbol{w}_{1}, \boldsymbol{w}_{2}, \boldsymbol{w}_{3}\right\}$, the object frame $\mathscr{B}_{o}:\left\{O_{\mathscr{B}}^{o}, \boldsymbol{b}_{1}^{o}, \boldsymbol{b}_{2}^{o}, \boldsymbol{b}_{3}^{o}\right\}$, the body frames $\mathscr{B}_{i}$ : $\left\{O_{\mathscr{B}}^{i}, b_{1}^{i}, b_{2}^{i}, b_{3}^{i}\right\}$ for each robot $i=1 \ldots n$, and the contact frame $\mathscr{C}_{i}:\left\{O_{c}^{i}, \boldsymbol{o}_{i}, \boldsymbol{t}_{i}, \boldsymbol{n}_{i}\right\}$ for each contact point $i=1 \ldots n$, placed where the adhesive mechanism of each passive tool is attached to the grasped object. The points $O_{\mathscr{B}}^{o}$ and $O_{\mathscr{B}}^{i}$, $i=1 \ldots n$, coincide with the CoMs of the object and the robots, respectively. The normal vectors $\boldsymbol{n}_{i}$ in the contact frames point to the out of the object (Fig. II).

The position of $O_{\mathscr{B}}^{i}$ in $\mathscr{W}$ is indicated with $\boldsymbol{p}_{i}=$ $\left[p_{1}^{i} p_{2}^{i} p_{3}^{i}\right]^{\top} \in \mathbb{R}^{3}$, and $m_{i} \in \mathbb{R}^{+}$and $J_{i} \in \mathbb{R}^{3 \times 3}$ are the mass and inertia matrix of each robot, respectively. The orthogonal matrix $R_{i} \in S O(3)$ represents the rotation from $\mathscr{W}$ to $\mathscr{B}_{i}$. When needed, we consider the RPY parametrization of $R_{i}$, i.e., $\boldsymbol{\eta}_{\boldsymbol{i}}:=\left[\phi_{i} \theta_{i} \psi_{i}\right]^{\top} \in \mathbb{R}^{3}$, where $\phi, \theta, \psi$ are the roll, pitch and yaw angles, respectively. The angular velocity of $\mathscr{B}_{i}$ with respect to $\mathscr{W}$, expressed in $\mathscr{B}_{i}$, is denoted with $\boldsymbol{\omega}_{i} \in \mathbb{R}^{3}$.

The magnitude of the total thrust acting along the $\boldsymbol{b}_{3}^{i}$ direction, denoted with $\lambda_{i} \in \mathbb{R}^{+}$, and the total moment expressed in $\mathscr{B}_{i}$, denoted with $\boldsymbol{u}_{i}=\left[\begin{array}{lll}u_{1}^{i} & u_{2}^{i} & u_{3}^{i}\end{array}\right]^{\top}$, are the four control inputs of each robot. The $i$-th VTOL's dynamic equation of motion in the operational space is

$$
M_{i}\left(\boldsymbol{x}_{\boldsymbol{i}}\right) \ddot{\boldsymbol{x}}_{\boldsymbol{i}}+\boldsymbol{b}_{\boldsymbol{i}}\left(\boldsymbol{x}_{\boldsymbol{i}}, \dot{\boldsymbol{x}}_{\boldsymbol{i}}\right)+\boldsymbol{g}_{\boldsymbol{i}}=\boldsymbol{h}_{\boldsymbol{i}}+\boldsymbol{l}_{\boldsymbol{i}}
$$

where $\boldsymbol{x}_{\boldsymbol{i}}=\left[\boldsymbol{p}_{\boldsymbol{i}}^{\top} \boldsymbol{\eta}_{\boldsymbol{i}}^{\top}\right]^{\top} \in \mathbb{R}^{6}$ is the robot pose, $\dot{\boldsymbol{x}}_{\boldsymbol{i}}$ and $\ddot{\boldsymbol{x}}_{\boldsymbol{i}}$ are its velocity and acceleration. The matrix $M_{i}=$ Blockdiag $\left(m_{i} I_{3 \times 3}, M_{\boldsymbol{\eta}, i}\left(\boldsymbol{\eta}_{\boldsymbol{i}}\right)\right) \in \mathbb{R}^{6 \times 6}$ is the inertia matrix, where $M_{\boldsymbol{\eta}, \boldsymbol{i}}\left(\boldsymbol{\eta}_{\boldsymbol{i}}\right)=E_{i}^{\top} J_{i} E_{i} \in \mathbb{R}^{3 \times 3}$. The vector $\boldsymbol{b}_{\boldsymbol{i}}=$ $\left[\mathbf{0}_{1 \times 3}\left(C_{i}\left(\boldsymbol{\eta}_{\boldsymbol{i}}, \dot{\boldsymbol{\eta}}_{\boldsymbol{i}}\right) \dot{\boldsymbol{\eta}}_{\boldsymbol{i}}\right)^{\top}\right]^{\top}$ is the Coriolis/centripetal effect, where $C_{\boldsymbol{\eta}, i}\left(\boldsymbol{\eta}_{\boldsymbol{i}}, \dot{\boldsymbol{\eta}}_{\boldsymbol{i}}\right)=E_{i}^{\top} J_{i} \dot{E}_{i}+E_{i}^{\top} S\left(E_{i} \dot{\boldsymbol{\eta}}_{i}\right) J_{i} E_{i} \in \mathbb{R}^{3 \times 3}$, and $E_{i}\left(\boldsymbol{\eta}_{\boldsymbol{i}}\right) \in \mathbb{R}^{3 \times 3}$ is a matrix that relates $\boldsymbol{\omega}_{i}$ to $\dot{\boldsymbol{\eta}}_{i}$ as $\boldsymbol{\omega}_{i}=$ $E_{i}\left(\boldsymbol{\eta}_{\boldsymbol{i}}\right) \dot{\boldsymbol{\eta}}_{i}$, and $S(\bullet)$ denotes the skew-symmetric matrix corresponding to a generic vector $\bullet$. The vector $\boldsymbol{g}_{\boldsymbol{i}}=$ $\left[\left(m_{i} g \boldsymbol{w}_{3}\right)^{\top} \mathbf{0}_{1 \times 3}\right]^{\top} \in \mathbb{R}^{6}$ is the gravity vector in which $g$ is the gravity acceleration constant.

The control input vector of each VTOL, expressed in $\mathscr{W}$, is $\boldsymbol{h}_{\boldsymbol{i}}=\left[\begin{array}{ll}\boldsymbol{f}_{i}^{\top} & \boldsymbol{\tau}_{i}^{\top}\end{array}\right]^{\top} \in \mathbb{R}^{6}$, where $\boldsymbol{f}_{i}=\lambda_{i} R_{i}\left[\begin{array}{lll}0 & 0 & 1\end{array}\right]^{\top} \in \mathbb{R}^{3}$ and $\boldsymbol{\tau}_{i}=R_{i} \boldsymbol{u}_{i} \in \mathbb{R}^{3}$. The load effect of the carried object on each VTOL is expressed by $\boldsymbol{l}_{i}=\left[\begin{array}{ll}\boldsymbol{d}_{i}^{\top} & \mathbf{0}_{1 \times 3}\end{array}\right]^{\top} \in \mathbb{R}^{6}$. Note that using the spherical joint at the CoM of the VTOL, we can assume the forces from the object result in negligible torques on the CoM of the VTOL, and we can consider the orientation of each VTOL independent from the orientation of the object [8], a part from the presence of spherical joint limits that will be carefully considered in our control framework.

Let $m_{o} \in \mathbb{R}$ and $\boldsymbol{J}_{o} \in \mathbb{R}^{3 \times 3}$ be the mass and inertia matrix of the object, and matrix $R_{o} \in \mathbb{R}^{3 \times 3}$ represent the rotation matrix from $\mathscr{W}$ to $\mathscr{B}_{o}$. The dynamic equation of motion for the object carried by the team of VTOLs is

$$
M_{o}(\boldsymbol{x}) \ddot{\boldsymbol{x}}+\boldsymbol{b}_{o}(\boldsymbol{x}, \dot{\boldsymbol{x}})+\boldsymbol{g}_{\boldsymbol{o}}=-G \boldsymbol{d}
$$

where $\boldsymbol{x}=\left[\boldsymbol{p}_{o}^{\top} \boldsymbol{\eta}_{o}^{\top}\right]^{\top}$ is the object's CoM pose, $M_{o}(\boldsymbol{x}) \in$ $\mathbb{R}^{6 \times 6}$ and $\boldsymbol{b}_{\boldsymbol{o}}(\boldsymbol{x}, \dot{\boldsymbol{x}}) \in \mathbb{R}^{6}$ are the inertial matrix and Coriolis/centripetal term, $\boldsymbol{g}_{\boldsymbol{o}}(\boldsymbol{x})$ is the gravity vector, $\boldsymbol{d}=$ $\left[\boldsymbol{d}_{\mathbf{1}}^{\top} \ldots \boldsymbol{d}_{\boldsymbol{n}}^{\top}\right]^{\top} \in \mathbb{R}^{3 n}$ is a vector containing forces applied by all the VTOLs to the object, and $G=\left[G_{1} \ldots G_{n}\right] \in \mathbb{R}^{6 \times 3 n}$ is the grasp matrix [21] in which each $G_{i}$ is

$$
G_{i}=\left[\begin{array}{ll}
\boldsymbol{I}_{3 \times 3} & S\left(R_{o}^{\top} \boldsymbol{r}_{\boldsymbol{i}}\right)^{\top}
\end{array}\right]^{\top}
$$

where $\boldsymbol{r}_{i} \in \mathbb{R}^{3}$ is a vector describing the point of application of the total thrust of the $i$-th VTOL, i.e., the fixed distance of $O_{\mathscr{B}}^{i}$, the CoM of each VTOL, from $O_{\mathscr{B}}^{o}$, the CoM of the object, in $\mathscr{B}_{o}$. Therefore, notice that the point included in the grasp matrix is the CoM of the $i$-th VTOLs rather than the contact point $O_{c}^{i}$ (where the adhesive mechanism of the passive tool is attached to the transported object). The position of $O_{\mathscr{B}}^{i}$, in $\mathscr{W}$, as depicted in Fig.II (a) is

$$
\boldsymbol{p}_{i}=\boldsymbol{p}_{o}+R_{o} \boldsymbol{r}_{\boldsymbol{i}} .
$$

The force applied to the object by the $i$-th robot, i.e., $\boldsymbol{d}_{\boldsymbol{i}}$, is the opposite of the first 3 components of $l_{i}$ in (1), and can be written as

$$
\boldsymbol{d}_{\boldsymbol{i}}=m_{i}\left(\ddot{\boldsymbol{p}}_{o}+\ddot{R}_{o} \boldsymbol{r}_{\boldsymbol{i}}+g \boldsymbol{w}_{3}\right)-\boldsymbol{f}_{\boldsymbol{i}}
$$

Substituting (5) in (2), we may express the whole system dynamic equation of motion as follows.

$$
M(\boldsymbol{x}) \ddot{\boldsymbol{x}}+\boldsymbol{b}(\boldsymbol{x}, \dot{\boldsymbol{x}})+\boldsymbol{g}=G \boldsymbol{f}
$$

where $M(\boldsymbol{x}) \in \mathbb{R}^{6 \times 6}, \boldsymbol{b}(\boldsymbol{x}, \dot{\boldsymbol{x}}) \in \mathbb{R}^{6}$, and $\boldsymbol{g} \in \mathbb{R}^{6}$ are the inertial matrix, Coriolis/centripetal term, and gravity vector; $\boldsymbol{f}=\left[\boldsymbol{f}_{\mathbf{1}}^{\top} \ldots \boldsymbol{f}_{\boldsymbol{n}}^{\top}\right]^{\top} \in \mathbb{R}^{3 n}$ is a vector containing total thrust (control) forces of all robots.

In this paper, we assume that a grasp planner arranges the contact points, such that the grasp matrix $G$ is full row rank, 
i.e., $\operatorname{Rank}(G)=6$. This is always true for more than two non-collinear contact points [21].

\section{B. Master Model}

The gravity compensated dynamic model of the haptic interface with $n_{m}$ DoFs can be described as

$$
M_{m}\left(\boldsymbol{x}_{m}\right) \ddot{\boldsymbol{x}}_{m}+C_{m}\left(\boldsymbol{x}_{m}, \dot{\boldsymbol{x}}_{m}\right) \dot{\boldsymbol{x}}_{m}=\boldsymbol{f}_{\boldsymbol{h}}+\boldsymbol{f}_{\boldsymbol{c}}
$$

where $\boldsymbol{x}_{m} \in \mathbb{R}^{n_{m}}$ is the configuration vector of the interface, $M_{m}\left(\boldsymbol{x}_{m}\right) \in \mathbb{R}^{n_{m} \times n_{m}}$, is the inertia matrix, $C_{m}\left(\boldsymbol{x}_{m}, \dot{\boldsymbol{x}}_{m}\right) \in$ $\mathbb{R}^{n_{m} \times n_{m}}$ is the Coriolis/centripetal matrix, and $\boldsymbol{f}_{\boldsymbol{h}} \in \mathbb{R}^{n_{m}}$ and $f_{c} \in \mathbb{R}^{3}$ are the human and control forces, respectively [22].

\section{System Constraints}

We consider the following constraints in our model:

- there are spherical joint limits, i.e., the set of allowed rotation of the spherical joints is realistically limited,

- each VTOL has a limited thrust, i.e., the total thrust magnitude for each VTOL must be comprised between a minimum and a maximum value (both positive),

- the object is fragile, i.e., there is a maximum normal force applicable to the object at the contact point,

- bounded strength of the adhesion mechanism at the contact point, i.e., the internal wrench at the contact point must be bounded in order to either keep the adhesion to the surface or to not break the gripper, depending on the contact method used.

\section{CONTROL SYSTEM}

In the proposed bilateral teleoperation control scheme for cooperative aerial manipulation, the desired object pose (position and orientation) is generated online by the human operator through a haptic device. The desired object pose and the current state of the object are then sent to the object pose controller that computes the desired total wrench that, if perfectly executed by the team of VTOLs, would let the object asymptotically track the desired object pose. The desired total wrench is then passed to the force allocation algorithm that, exploiting the system redundancy, computes a set of $n$ desired forces to be applied at the $n$ VTOL CoMs. The collective execution of these forces produces the desired total wrench and, at the same time, satisfies all the set of constraints, which have been introduced in Sec II$\mathrm{C}$ and will be formally defined in Sec III-B. Finally, the desired force corresponding to each VTOL is passed to the corresponding attitude-and-thrust controller (one for each VTOL) that controls the moment and thrust magnitude of the VTOL in order to track the desired force to be applied at the VTOL CoM.

On the backward channel of the bilateral teleoperation scheme, the human receives a force feedback that depends on the inertia of the whole system and on a repulsive viscoelastic virtual force generated with the purpose of letting the operator feel the obstacles in the environment.

In the following we explain in detail all the components of the control scheme which are summarized in Figure III.

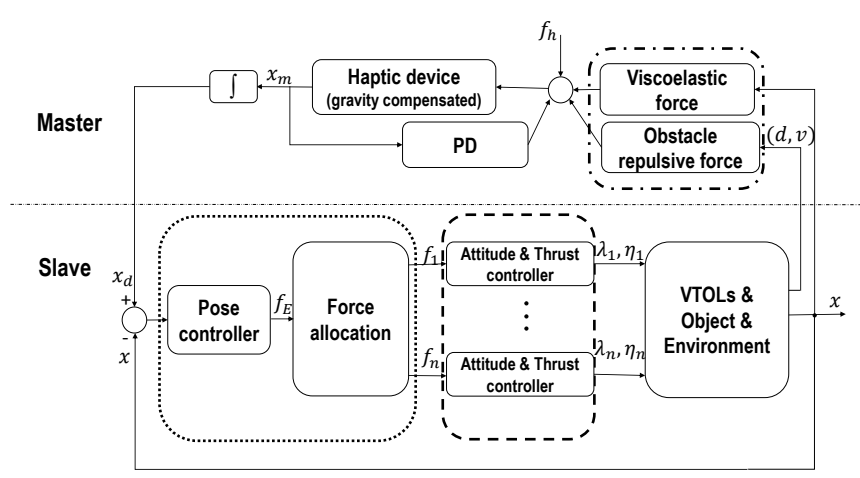

Fig. 3: The bilateral teleoperation scheme for cooperative aerial manipulation: the object pose controller and force allocation algorithm (dotted rectangle), the VTOLs' thrust-and-attitude controller (dashed rectangle), and the haptic feedback (dash-dotted rectangle).

\section{A. Object Pose Controller}

The object pose controller generates a desired wrench $\boldsymbol{f}_{\boldsymbol{E}} \in \mathbb{R}^{6}$ to balance the object dynamics, cancel possible external disturbances, and track the desired pose trajectory $\boldsymbol{x}_{d} \in \mathbb{R}^{6}$ commanded by the human operator. Let $\hat{M}, \hat{\boldsymbol{b}}$, and $\hat{\boldsymbol{g}}$ be the estimation of $M, \boldsymbol{b}$, and $\boldsymbol{g}$, respectively. The pose control law is defined as

$$
\left\{\begin{array}{l}
\boldsymbol{f}_{E}=\hat{M}(\boldsymbol{x}) \boldsymbol{\mu}+\hat{\boldsymbol{b}}(\boldsymbol{x}, \dot{\boldsymbol{x}})+\hat{\boldsymbol{g}}(\boldsymbol{x}) \\
\boldsymbol{\mu}=K_{P} \tilde{\boldsymbol{x}}+K_{D} \dot{\tilde{\boldsymbol{x}}}
\end{array}\right.
$$

where $\tilde{\boldsymbol{x}}=\boldsymbol{x}_{d}-\boldsymbol{x}$ is the pose error, and $K_{P}, K_{D} \in \mathbb{R}^{6 \times 6}$ are the diagonal control gain matrices with positive entries.

\section{B. Force Allocation Algorithm}

The force allocation algorithm exploits the redundancy of the system in order to generate the desired wrench and at the same time satisfy the system constraints which are formally derived in the following.

Let $\left[f_{i, b}^{x} f_{i, b}^{y} f_{i, b}^{z}\right]^{\top}=\lambda_{i} R_{o}^{\top} R_{i}\left[\begin{array}{lll}0 & 0 & 1\end{array}\right]^{\top} \in \mathbb{R}$ be the force components produced by each VTOL at $O_{\mathscr{B}}^{i}$, expressed in $\mathscr{B}_{o}$, and $\gamma \in\left(0, \frac{\pi}{2}\right)$ be half of the aperture angle of the cone that defines the maximum rotation allowed by the spherical joint. The force components must comply with the following inequality

$$
f_{i, b}^{z}>\vartheta \sqrt{\left(f_{i, b}^{x}\right)^{2}+\left(f_{i, b}^{y}\right)^{2}}
$$

where $\vartheta=\tan (\gamma) \in \mathbb{R}^{+}$describes the possible cone of rotation. This constraint can be rewritten as

$$
\left\|\boldsymbol{f}_{\boldsymbol{i}}\right\| \leq \beta f_{i, b}^{z}
$$

where $\beta=\frac{\sqrt{1+\vartheta^{2}}}{\vartheta} \in \mathbb{R}^{+}$.

The norm of each force vector, applied by each VTOL to the spherical joint, must comply with the actuator allowed range

$$
f_{p}^{\min } \leq\left\|\boldsymbol{f}_{\boldsymbol{i}}\right\| \leq f_{p}^{\max }
$$

where $f_{p}^{\max } \in \mathbb{R}^{+}$is the upper bound specified by the motors power, and $f_{p}^{\text {min }} \in \mathbb{R}^{+}$is the lower bound, demanded by the motors to stay operational. 
To avoid damaging the object, the component of the force along $-\boldsymbol{n}_{i}$ must be less than a threshold

$$
\boldsymbol{f}_{\boldsymbol{i}}^{\top} \boldsymbol{n}_{i} \geq-f_{m}^{n}
$$

where $f_{m}^{n} \in \mathbb{R}^{+}$depends on the fragility of the object.

Now let $\mu_{s}, \mu_{t} \in \mathbb{R}^{+}$be the linear and rotational static friction coefficients between the adhesive disk and the object surface. The adhesive contact force $\boldsymbol{f}_{i}^{m}$ can be expressed as

$$
\boldsymbol{f}_{i}^{m}=-k_{i}^{m} \boldsymbol{n}_{i}
$$

where $k_{i}^{m} \in \mathbb{R}^{+}$is a constant related to the strength of the adhesion mechanism. We indicate with $\tau_{c}^{i}=S\left(\boldsymbol{r}_{b}^{i}\right) \boldsymbol{f}_{\boldsymbol{i}}$ the torques applied to the adhesive disc of radius $r_{d}^{i} \in \mathbb{R}^{+}$, expressed in contact frame, as $\boldsymbol{\tau}_{c}^{i}=\left[\tau_{o}^{i}, \tau_{t}^{i}, \tau_{n}^{i}\right]^{\top}$. Where $\boldsymbol{r}_{\boldsymbol{b}}^{i}$ is vector showing the fixed distance of each robot's CoM, that is, the spherical joint place, from the contact point, which is the center of the adhesive disk (Fig. II (b)). To avoid breaking the contact, the normal force component magnitude applied at each contact point must be less than the adhesive force magnitude. Furthermore, the total normal force at each contact point is the sum of the adhesive force and the normal component of the force applied by each VTOL. Thus, in each contact point to avoid planar slippage, the magnitude of linear static friction force must be greater than the magnitude of the planar force, and to avoid rotational slippage, the magnitude of the rotational friction torque must be greater than the magnitude of the normal torque component $\left(\tau_{n}^{i}\right)$. Moreover, to avoid breaking the contact due to other two torque components $\left(\tau_{o}^{i}\right.$ and $\tau_{t}^{i}$ ), the sum of their magnitudes must not be greater than the adhesive torque magnitude (the adhesive force result) around any pivot point at the disk edge. The constraints on forces and torques to avoid breaking the contact are summarized as

$$
\left\{\begin{array}{l}
\sqrt{\left(\boldsymbol{f}_{i}^{\top} \boldsymbol{t}_{i}\right)^{2}+\left(\boldsymbol{f}_{i}^{\top} \boldsymbol{o}_{i}\right)^{2}} \leq \mu_{s} \mid\left(k_{i}^{m}+\left(\boldsymbol{f}_{i}^{\top} \boldsymbol{n}_{i}\right) \mid\right. \\
\boldsymbol{f}_{i}^{\top} \boldsymbol{n}_{i} \leq k_{i}^{m} \\
\left\|\boldsymbol{\tau}_{i}^{n}\right\| \leq \mu_{t} \mid\left(k_{i}^{m}+\left(\boldsymbol{f}_{i}^{\top} \boldsymbol{n}_{i}\right) \mid\right. \\
\sqrt{\left(\boldsymbol{\tau}_{i}^{t}\right)^{2}+\left(\boldsymbol{\tau}_{i}^{o}\right)^{2}} \leq r_{d} k_{i}^{m}
\end{array}\right.
$$

The constraints (9),(10),(11), and (12) for all VTOLs can be summarized as $\chi(f)=\left[\chi_{1}^{\top}\left(f_{1}\right) \ldots \chi_{n}^{\top}\left(f_{n}\right)\right]^{\top} \leq 0$, where $\chi_{i}\left(f_{i}\right): \mathbb{R}^{3} \rightarrow \mathbb{R}^{8}$ is defined as follows

$$
\chi_{\boldsymbol{i}}\left(\boldsymbol{f}_{\boldsymbol{i}}\right)=\left[\begin{array}{c}
\left\|\boldsymbol{f}_{\boldsymbol{i}}\right\|-\beta f_{i, b}^{z} \\
\left\|\boldsymbol{f}_{\boldsymbol{i}}\right\|-f_{p}^{\max } \\
-\left\|\boldsymbol{f}_{\boldsymbol{i}}\right\|+f_{p}^{\min } \\
-\boldsymbol{f}_{\boldsymbol{i}}^{\top} \boldsymbol{n}_{i}-f_{m}^{n} \\
\sqrt{\left(\boldsymbol{f}_{i}^{\top} \boldsymbol{t}_{i}\right)^{2}+\left(\boldsymbol{f}_{i}^{\top} \boldsymbol{o}_{i}\right)^{2}-\mu_{s} \mid\left(k_{i}^{m}+\left(\boldsymbol{f}_{i}^{\top} \boldsymbol{n}_{i}\right) \mid\right.} \\
\boldsymbol{f}_{i}^{\top} \boldsymbol{n}_{i}-k_{i}^{m} \\
\left\|\boldsymbol{\tau}_{i}^{n}\right\|-\mu_{t} \mid\left(k_{i}^{m}+\left(\boldsymbol{f}_{i}^{\top} \boldsymbol{n}_{i}\right) \mid\right. \\
\sqrt{\left(\boldsymbol{\tau}_{i}^{t}\right)^{2}+\left(\boldsymbol{\tau}_{i}^{o}\right)^{2}}-r_{d} k_{i}^{m}
\end{array}\right]
$$

The problem of force allocation, is also subject to the equality constraints $\boldsymbol{\xi}(\boldsymbol{f})=0$ where $\boldsymbol{\xi}: \mathbb{R}^{3 n} \rightarrow \mathbb{R}^{6}$ is

$$
\boldsymbol{\xi}(\boldsymbol{f})=G \boldsymbol{f}-\boldsymbol{f}_{\boldsymbol{E}} .
$$

The force allocation problem can be seen as a programming problem to minimize the cost function

$$
J(\boldsymbol{f})=\frac{1}{2} \boldsymbol{f}^{\top} \boldsymbol{f}+\boldsymbol{\varepsilon}\left(\boldsymbol{f}-\boldsymbol{f}_{k-1}\right)^{\top}\left(\boldsymbol{f}-\boldsymbol{f}_{k-1}\right)
$$

where $J: \mathbb{R}^{3 n} \rightarrow \mathbb{R}$, and $\boldsymbol{f} \in \mathscr{F}$, where $\mathscr{F}$ is the feasible solution set defined by the inequality and equality constraints (13) and (14). The solution of the optimization problem in the previous time step is denoted with $\boldsymbol{f}_{k-1}$, and $\varepsilon \in \mathbb{R}$ is a positive scalar. The convex quadratic cost function (15) is a weighted sum of a minimal force objective and a term penalizing variation. $\varepsilon$ is a tuning knob directly proportional to the input smoothness, as that non negative term is minimized if $\boldsymbol{f}=\boldsymbol{f}_{k-1}$. In summary, the force allocation problem is

$$
\begin{array}{rl}
\boldsymbol{f}^{*}=\arg \min _{\boldsymbol{f}} & J(\boldsymbol{f}) \\
\text { s.t. } & \chi(\boldsymbol{f}) \leq 0 \\
& \boldsymbol{\xi}(\boldsymbol{f})=0 .
\end{array}
$$

Both $\chi(f)$ and $\xi(f)$ are affine functions of the optimization variable $\boldsymbol{f}$. That, along with $J(\boldsymbol{f})$ being convex quadratic, makes (16) a convex programming problem. There are several standard efficient methods to solve this problem, see, e.g., [23].

\section{Attitude-and-Thrust Controller}

The attitude-and-thrust controller generates the thrust and moment controls of the VTOL corresponding to the allocated desired force $f_{i}$ of each VTOL. For each VTOL, the thrust magnitude is directly controlled, and the yaw angle is set to follow the object yaw angle. As previously stated in Section II-A $f_{i}=\lambda_{i} R_{i}\left[\begin{array}{lll}0 & 0 & 1\end{array}\right]^{\top}$, thus the desired thrust $\lambda_{d}^{i} \in \mathbb{R}^{+}$and the desired rotation matrix $R_{d}^{i} \in S O(3)$ can be defined as

$$
\left\{\begin{array}{l}
\lambda_{d}^{i}=\boldsymbol{f}_{i}^{\top} R_{i}\left[\begin{array}{lll}
0 & 0 & 1
\end{array}\right]^{\top} \\
\frac{\boldsymbol{f}_{i}}{\left\|\boldsymbol{f}_{i}\right\|}=R_{d}^{i}\left[\begin{array}{lll}
0 & 0 & 1
\end{array}\right]^{\top} .
\end{array}\right.
$$

A wide range of attitude controllers, such as the one presented in [24] can be used to produce $\tau_{i}$. Let us define the orientation matrix error as

$$
e_{R}^{i}=\frac{1}{2} S^{-}\left(R_{d}^{i} R_{i}-R_{i}^{\top} R_{d}^{i}\right)
$$

where $S^{-}(\bullet)$ is the inverse operation of $S(\bullet)$, the rotation error, corresponding to the rotation matrix $R_{d}^{\top} R$, is

$$
e_{\omega}^{i}=\omega_{i}-R_{i}^{\top} R_{d}^{i} \omega_{d}^{i}
$$

where $\boldsymbol{\omega}_{d}^{i} \in \mathbb{R}^{3}$ is the desired angular velocity, and the attitude control law $\tau_{i}$ is

$$
\tau_{i}=-K_{R} e_{R}^{i}-K_{\omega} e_{\omega}^{i}+\omega_{i} \times J_{i} \omega_{i}
$$

in which $K_{R}, K_{\omega} \in \mathbb{R}^{3 \times 3}$ are diagonal matrices with positive elements. Interested readers are encouraged to see [24] for the stability proof of the controller.

\section{Haptic feedback}

The human operator applies $\boldsymbol{f}_{h}$ to the haptic device, changing its position $\boldsymbol{x}_{m}$. in order to cope with the limited workspace of the haptic device, the integrated over time $\boldsymbol{x}_{m}$ is given to the slave as reference pose trajectory $x_{d}$. The control force for the master device $f_{c}$ is computed as

$$
\boldsymbol{f}_{c}=\boldsymbol{f}_{m}+\boldsymbol{f}_{o}+\boldsymbol{f}_{e} .
$$


The term $\boldsymbol{f}_{m}$, which is intended to bring back the haptic probe to its centered position in order to avoid drifting of the slave, is defined as

$$
\boldsymbol{f}_{m}=-K_{h} \operatorname{sat}\left(\boldsymbol{x}_{m}, \boldsymbol{x}_{m}^{\max }\right)-B_{h} \dot{\boldsymbol{x}}_{m}
$$

where $K_{h}, B_{h} \in \mathbb{R}^{3 \times 3}$ are diagonal gain matrices with positive entries, $\boldsymbol{x}_{m}^{\max } \in \mathbb{R}^{+}$is a scalar to limit the force, $\operatorname{sat}\left(\boldsymbol{x}_{m}, \boldsymbol{x}_{m}^{\max }\right)$ is a saturation function defined as

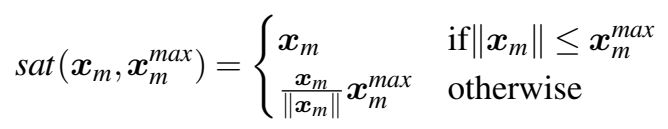

The first term in the rhs of (22) is to set the haptic probe in absence of external forces. The second term is a damping factor that prevents oscillatory behaviors of the device.

The repulsive force feedback $\boldsymbol{f}_{o}$ is meant to avoid collision with the obstacles in the environment and is based on the basic risk field (BRF) [25]

$$
\boldsymbol{f}_{o}=\left\{\begin{array}{ll}
0 & \text { if } \quad \frac{1+v}{d_{r e s}(d, v)} \leq 0 \\
f_{o}^{\max } \boldsymbol{n}_{o} & \text { if }\left\{\begin{array}{l}
d_{\text {res }}(d, v) \leq 0 \\
\frac{1+v}{d_{r e s}(d, v)} \geq \frac{1}{k_{o}}
\end{array}\right. \\
k_{o} \frac{1+v}{d_{r e s}(d, v)} n_{o} & \text { otherwise }
\end{array},\right.
$$

where $\boldsymbol{n}_{\boldsymbol{o}} \in \mathbb{R}^{3}$ is the unit vector representing the direction of the repulsive force, $d$ is distance from the obstacle, $v$ is the component of velocity toward the obstacle, $k_{o}$ is a gain to tune the sensitivity, $f_{o}^{\max }$ is the maximum force to be felt in haptic device, and the reserve avoidance distance $d_{r e s}$ is defined as

$$
d_{r e s}(d, v)=\left\{\begin{array}{lll}
\frac{2 a_{\max } d+v^{2}}{2 a_{\max }} & \text { if } & v \leq 0 \\
\frac{2 a_{\max } d-v^{2}}{2 a_{\max }} & \text { if } & v>0
\end{array},\right.
$$

where $a_{\max }$ is the maximum deceleration in the obstacle direction.

Finally, the term $f_{e}$ in (22) is intended to improve the tracking performance

$$
\boldsymbol{f}_{e}=K_{1} \tilde{\boldsymbol{x}}+K_{2} \dot{\tilde{\boldsymbol{x}}}
$$

where $\tilde{\boldsymbol{x}}=\boldsymbol{x}_{d}-\boldsymbol{x}, \dot{\tilde{\boldsymbol{x}}}=\dot{\boldsymbol{x}}_{d}-\dot{\boldsymbol{x}}$, and $K_{1}, K_{2} \in \mathbb{R}^{3 \times 3}$ are diagonal matrices with positive elements.

\section{Human/Hardware in the Loop Simulations}

Human/Hardware In the Loop (HIL) simulations have been performed in Simulink ${ }^{\circledR}$ using SimMechanics ${ }^{\mathrm{TM}}$ with a control loop of $1 \mathrm{~ms}$ for haptic interface, $10 \mathrm{~ms}$ for quadrotors thrust and attitude controller, and the force allocation optimization solver. Sample time enforcement was carefully measured.

In order to solve the optimization problem we adopted the open source tool Optpp [26] and in particular an instance of OptNIPS problem, namely an implementation of Newton nonlinear interior-point method with analytic Hessian information. Pre-computed analytic representations of the first and second derivatives of the cost function and constraints were obtained by means of Matlab ${ }^{\circledR}$ symbolic and code

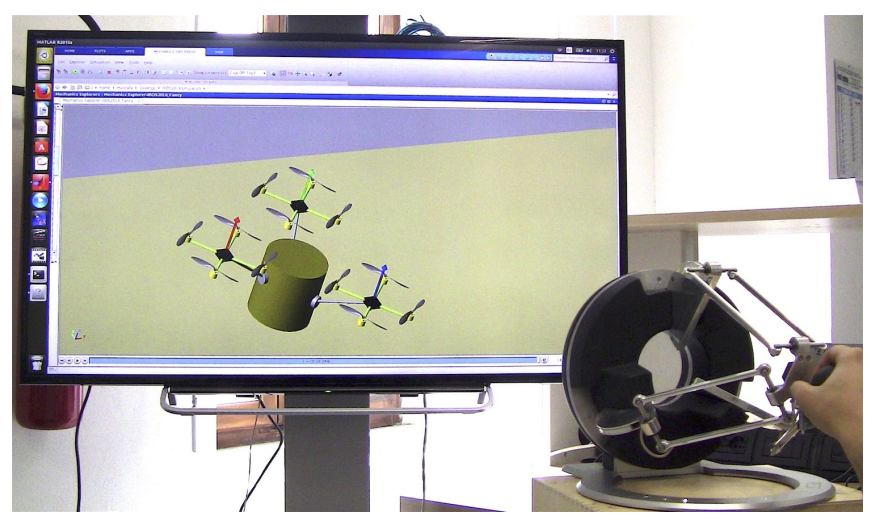

Fig. 4: HIL simulation setup.

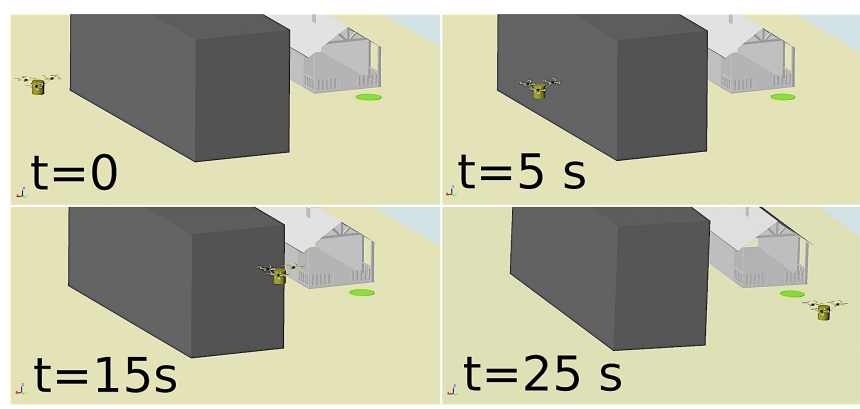

Fig. 5: HIL simulation scenario.

generation toolboxes, which produced $\mathrm{C}++$ code eventually bound to Simulink ${ }^{\circledR}$ via a C++ level-2 S-Function.

The HIL simulation scenario is as follows. The VTOL team consists of three quadrotors carrying a barrel in the virtual environment, and a human operator drives them via a 3-DoF actuated haptic device (Fig. 4). The initial position of the grasped object is $(0,0,0)$, while the ground is considered a flat surface 1 meter below the object, and he target point is $(-7 \mathrm{~m}, 0,0)$. There is a cube shape obstacle of size $(3 \mathrm{~m}, 6 \mathrm{~m}, 3 \mathrm{~m})$ centered at $(-2 \mathrm{~m}, 0,0)$. The mission is complete when the object's CoM is closer than $12 \mathrm{~cm}$ to the target point (Fig. 5). The translation of the haptic device is integrated to generate the reference trajectory for the object and the desired orientation set to be zero. The weight of each quadrotor, including the adhesive mechanism, is $0.7 \mathrm{~kg}$, and the object is a cylinder of $30 \mathrm{~cm}$ height, $24 \mathrm{~cm}$ diameter and $2.4 \mathrm{~kg}$ weight.

Locating the contact points exactly in the desired positions, which are determined by a grasp planner, is very difficult in our problem. Thus, we considered up to $10 \%$ uncertainty (with respect to the object size) in locating the contact points. A typical HIL simulation results are presented in Fig. 6, 7, 8, 9 in which the subject completed the task in 47.5 seconds.

Fig. 6(a) shows the commanded trajectory by the human operator and the actual path of the VTOL team carrying the object from the orthogonal view (xy-plane). Fig. 6(b) represents the normalized Euclidean norm of the position error (left y-axis) and attitude error (right y-axis). The position error norm is normalized by the team radius $r$, i.e., 

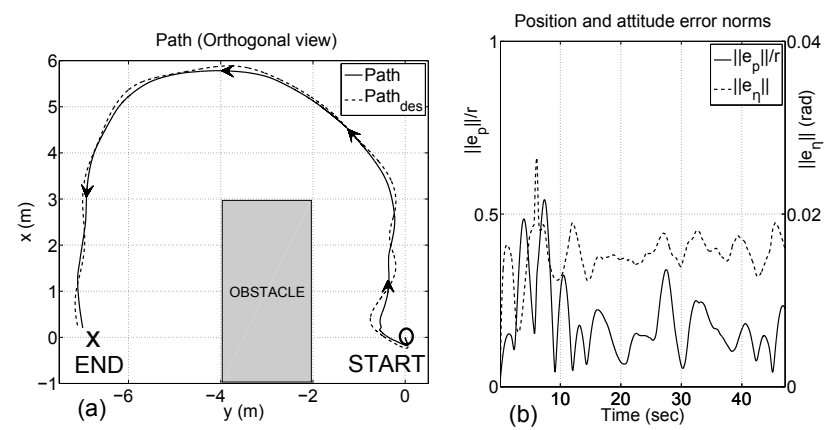

Fig. 6: (a) Desired and actual path of the VTOL team carrying the object. The path shows the orthogonal view of the object's CoM. (b) Euclidean norm of the position error (left y-axis), which is normalized by the radius size of the team. Euclidean norm of the attitude error (right y-axis).
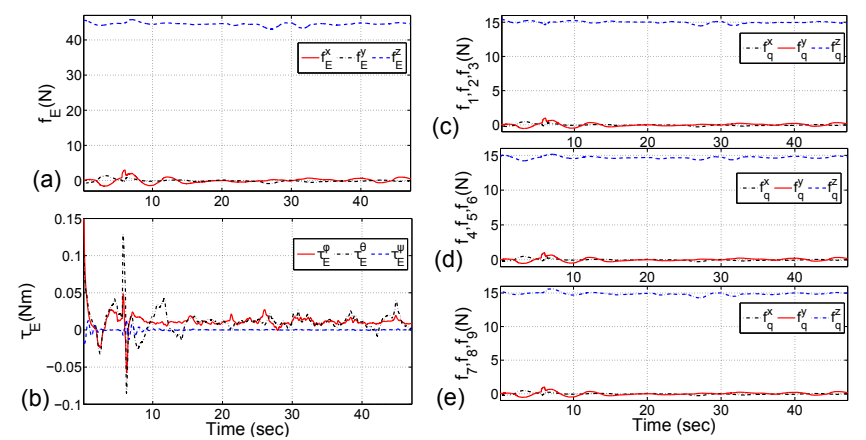

Fig. 7: (a) Object pose controller output forces (the first three elements of $\boldsymbol{f}_{\boldsymbol{E}}$ ). (b) Object pose controller output torques (the last three elements of $\left.f_{E}\right)$. (c, d, e) The allocated force vectors to the three quadrotors.

the circumambient sphere radius, to show the the control system efficiency. It is evident that the position error norm is smaller than half of the $r$, where $r=60 \mathrm{~cm}$. And the small error of the attitude norm is due to the uncertainty in the grasp matrix.

Fig. 7(a,b) depicts the object pose controller output $\left(\boldsymbol{f}_{\boldsymbol{E}}\right)$ to follow the user commanded trajectory, where the force elements are shown in Fig. 7(a) and the torque elements in Fig. 7(b). The force allocation algorithm generates the desired vector forces of each quadrotor, that is shown in Fig. 7(c, d, e).

To generate the force allocated to each quadrotor, attitude and thrust controller controls the attitude of quadrotors Fig. 8(a, c, e). Then, the thrust of four motors are calculated and applied to the motors to achieve the desired attitude and thrust Fig. 8(b, d, f). As it can be seen, due to the appropriate controller gains, the thrust of the motors are reasonable both in value and rate.

At the master side, the position of the haptic probe $\left(\boldsymbol{x}_{\boldsymbol{m}}\right)$ is shown in Fig. 9(b), and the corresponding force $\left(f_{m}\right)$, which is keeping the haptic probe at the center to avoid vehicle drifting, due to the integral action, which is intended to cope with the limited workspace of the haptic device, is shown in Fig. 9(b). The force feedback to improve the tracking performance $\left(f_{e}\right)$ in correspondence to the position error in tracking the desired trajectory is shown in Fig. 9(c), and
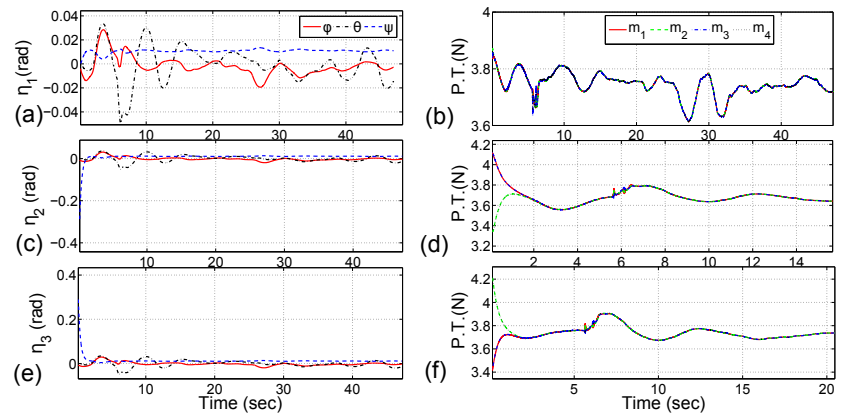

Fig. 8: (a, c, e) The attitude of VTOLs to produce their own allocated force vectors. (b, d, f) The thrust of four motors for each quadrotor to achieve the desired thrust and the attitude.
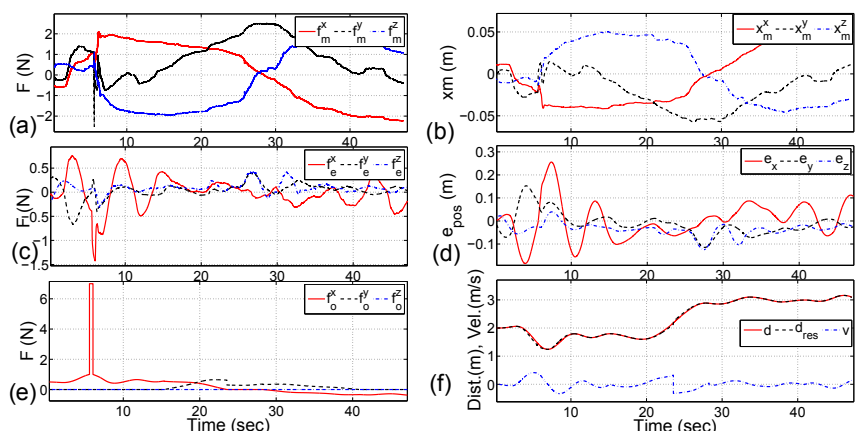

Fig. 9: (a) Centering force at the master side $\left(\boldsymbol{f}_{m}\right)$, (b) the position of the haptic probe $\left(\boldsymbol{x}_{m}\right)$. (c) Force feedback to improve the tracking performance $\left(f_{e}\right)$. (d) Position error in trajectory tracking. (e) The repulsive force feedback $\left(f_{o}\right)$. (e) Distance $(d)$, reserve avoidance distance $\left(d_{r e s}\right)$, and the velocity $(v)$ towards the obstacle.

the position error in 9 (d) represents the high performance of the tele-operated system with the maximum position error less than $30 \mathrm{~cm}$ during the flight time. The repulsive force feedback $\left(f_{o}\right)$ to avoid the obstacle is shown in Fig. 9(e), which is calculated according to the distance $(d)$, reserve avoidance distance $\left(d_{r e s}\right)$, and the velocity $(v)$ towards the obstacle Fig. 9(f).

In order to study the haptic feedback effect, we asked 10 subjects to do the HIL experiment once with haptic feedback and once without haptic feedback. The subject were able to see the virtual environment from a single fixed camera in the environment, thus did not have a full view of the VTOLs, due to the existence of the big obstacle in the environment. Using haptic feedback, $10 \%$ of the subjects experienced failure by colliding the object, while without haptic feedback $20 \%$ of the subjects had the incomplete task, due to the collision with the obstacle. For 10 successful trials, the time to complete the task $\left(t_{f}\right)$ is shorter with haptic feedback than without it, and also the tracking performance (in term of $\frac{1}{t_{f}} \int_{0}^{t_{f}}\|\tilde{x}\| d t$ ) is smaller for trials with haptic feedback than without haptic feedback. The average and standard deviation of the time to complete the task and tracking performance are summarized in Table I. 
TABLE I: Time to complete the task and tracking performance in HIL simulation study for ten subjects.

\begin{tabular}{lll}
\hline & With haptic feedback & Without haptic feedback \\
\hline Time to complete the task Avg.(Std.) sec & $26.88(5.21)$ & $27.82(3.92)$ \\
Tracking performance Avg.(Std.) m & $0.134(0.038)$ & $0.172(0.038)$ \\
\hline
\end{tabular}

\section{CONCLUSION}

In this paper a bilateral tele-operation scheme for cooperative aerial manipulation is presented. A human operator drives a team of VTOLs holding an object, which is grasped in advance, receives a force feedback based on the system state, i.e., position tracking error, repulsive force to avoid obstacles. To simplify mechanical design, each UAV equipped with a passive adhesive tool connected via spherical joint to the vehicle. The granted redundancy, in case of more than two robots, is used to optimally allocate among team members, the forces to track object pose reference. The online quadratic programming problem solves the allocation trading between force magnitude and signal smoothness, while enforcing system constraints. An attitude and thrust controller regulates the motor thrusts and attitude of each vehicle to meet the desired allocated force. A human/hardware in the loop simulation study showed the efficiency of the proposed scheme and the importance of haptic feedback in reducing the time to complete the task and also enhancing the tracking performance.

Future development planning includes i) implementation with real robots, using onboard equipment camera-like /bearing sensors and distributed estimation control schemes as, e.g., in [27], ii) being able to cope with an unknown load and non-perfect knowledge of the contact point position using sensor-based calibration methods, similar to the ones in [28] and distributed estimation, as in [29], and iii) address the grasp planning problem explicitly.

\section{REFERENCES}

[1] P. E. Pounds, D. R. Bersak, and A. M. Dollar, "Stability of smallscale uav helicopters and quadrotors with added payload mass under pid control," Autonomous Robots, vol. 33, no. 1-2, pp. 129-142, 2012.

[2] G. Gioioso, M. Ryll, D. Prattichizzo, H. Bulthoff, and A. Franchi, "Turning a near-hovering controlled quadrotor into a $3 \mathrm{~d}$ force effector," in Robotics and Automation (ICRA), 2014 IEEE International Conference on, May 2014, pp. 6278-6284.

[3] G. Gioioso, M. Mohammadi, A. Franchi, and D. Prattichizzo, "A forcebased bilateral teleoperation framework for aerial robots in contact with the environment," in Robotics and Automation (ICRA), 2015 IEEE International Conference on, May 2015, pp. 318-324.

[4] K. Kondak, F. Huber, M. Schwarzbach, M. Laiacker, D. Sommer, M. Bejar, and A. Ollero, "Aerial manipulation robot composed of an autonomous helicopter and a 7 degrees of freedom industrial manipulator," in Robotics and Automation (ICRA), 2014 IEEE International Conference on, May 2014, pp. 2107-2112.

[5] "Eu collab. project ict-287617," http://www.arcas-project.eu.

[6] R. Ritz and R. D'Andrea, "Carrying a flexible payload with multiple flying vehicles," in Intelligent Robots and Systems (IROS), 2013 IEEE/RSJ International Conference on. IEEE, 2013, pp. 3465-3471.

[7] V. Parra-Vega, A. Sanchez, C. Izaguirre, O. Garcia, and F. RuizSanchez, "Toward aerial grasping and manipulation with multiple uavs," Journal of Intelligent \& Robotic Systems, vol. 70, no. 1-4, pp. 575-593, 2013.
[8] S. P. Hai-Nguyen Nguyen and D. Lee, "Aerial tool operation system using quadrotors as rotating thrust generators," in Intelligent Robots and Systems (IROS), 2015 IEEE/RSJ International Conference on. IEEE, 2015, p. In press.

[9] M. B. Srikanth, A. Soto, A. Annaswamy, E. Lavretsky, and J.-J. Slotine, "Controlled manipulation with multiple quadrotors," in AIAA Conf. on Guidance, Navigation and Control, 2011.

[10] G. Gioioso, A. Franchi, G. Salvietti, S. Scheggi, and D. Prattichizzo, "The flying hand: A formation of uavs for cooperative aerial telemanipulation," in Robotics and Automation (ICRA), 2014 IEEE International Conference on, May 2014, pp. 4335-4341.

[11] Q. Jiang and V. Kumar, "The inverse kinematics of cooperative transport with multiple aerial robots," Robotics, IEEE Transactions on, vol. 29, no. 1, pp. 136-145, Feb 2013.

[12] K. Sreenath and V. Kumar, "Dynamics, control and planning for cooperative manipulation of payloads suspended by cables from multiple quadrotor robots," $r n$, vol. 1, no. r2, p. r3, 2013.

[13] H. Yang and D. Lee, "Hierarchical cooperative control framework of multiple quadrotor-manipulator systems," in Robotics and Automation (ICRA), 2015 IEEE International Conference on. IEEE, 2015, pp. 4656-4662.

[14] A. Franchi, C. Secchi, H. I. Son, H. H. Bulthoff, and P. R. Giordano, "Bilateral teleoperation of groups of mobile robots with time-varying topology," IEEE Transactions on Robotics, vol. 28, no. 5, pp. 10191033, Oct 2012.

[15] P. Griffiths and R. B. Gillespie, "Shared control between human and machine: haptic display of automation during manual control of vehicle heading," in Haptic Interfaces for Virtual Environment and Teleoperator Systems, 2004. HAPTICS '04. Proceedings. 12th International Symposium on, March 2004, pp. 358-366.

[16] M. Kovač, J. Germann, C. Hürzeler, R. Y. Siegwart, and D. Floreano, "A perching mechanism for micro aerial vehicles," Journal of MicroNano Mechatronics, vol. 5, no. 3-4, pp. 77-91, 2009.

[17] H. Tsukagoshi, M. Watanabe, T. Hamada, D. Ashlih, and R. Iizuka, "Aerial manipulator with perching and door-opening capability," in Robotics and Automation (ICRA), 2015 IEEE International Conference on. IEEE, 2015, pp. 4663-4668.

[18] N. Wiltsie, M. Lanzetta, and K. Iagnemma, "A controllably adhesive climbing robot using magnetorheological fluid," in Technologies for Practical Robot Applications (TePRA), 2012 IEEE International Conference on. IEEE, 2012, pp. 91-96.

[19] A. Parness, Micro-Structured Adhesives for Climbing Applicationjs. Stanford University, 2010.

[20] E. W. Hawkes, H. Jiang, and M. R. Cutkosky, "Three-dimensional dynamic surface grasping with dry adhesion," The International Journal of Robotics Research, p. 0278364915584645, 2015.

[21] D. Prattichizzo and J. C. Trinkle, "Grasping," in Springer handbook of robotics. Springer, 2008, pp. 671-700.

[22] A. Franchi, C. Secchi, M. Ryll, H. H. Bülthoff, and P. R. Giordano, "Shared control: Balancing autonomy and human assistance with a group of quadrotor uavs," Robotics \& Automation Magazine, IEEE, vol. 19, no. 3, pp. 57-68, 2012.

[23] S. Boyd and L. Vandenberghe, Convex optimization. Cambridge university press, 2004.

[24] T. Lee, M. Leoky, and N. H. McClamroch, "Geometric tracking control of a quadrotor uav on se (3)," in Decision and Control (CDC), 2010 49th IEEE Conference on. IEEE, 2010, pp. 5420-5425.

[25] T. M. Lam, H. W. Boschloo, M. Mulder, and M. M. Van Paassen, "Artificial force field for haptic feedback in uav teleoperation," Systems, Man and Cybernetics, Part A: Systems and Humans, IEEE Transactions on, vol. 39, no. 6, pp. 1316-1330, 2009.

[26] J. Meza, R. Oliva, P. Hough, and P. Williams, "Opt++: An objectoriented toolkit for nonlinear optimization," ACM Transactions on Mathematical Software (TOMS), vol. 33, no. 2, p. 12, 2007.

[27] A. Franchi and P. Robuffo Giordano, "Decentralized control of parallel rigid formations with direction constraints and bearing measurements," in 51st IEEE Conf. on Decision and Control, Maui, HI, Dec. 2012, pp. 5310-5317.

[28] A. Censi, A. Franchi, L. Marchionni, and G. Oriolo, "Simultaneous maximum-likelihood calibration of odometry and sensor parameters," IEEE Trans. on Robotics, vol. 29, no. 2, pp. 475-492, 2013.

[29] A. Franchi, A. Petitti, and A. Rizzo, "Decentralized parameter estimation and observation for cooperative mobile manipulation of an unknown load using noisy measurements," in 2015 IEEE Int. Conf. on Robotics and Automation, Seattle, WA, May 2015, pp. 5517-5522. 